\title{
A new look at the proof of $K$-theoretic amenability for groups acting on trees
}

\author{
Pierre Julg
}

\begin{abstract}
We generalize the construction by Pytlik and Szwarc of uniformly bounded representations for free groups to groups acting on trees. We deduce a new version of the proof (by Alain Valette and the author, 1983) of the fact that locally compact groups acting on trees with amenable stabilizers are amenable in K-theory.
\end{abstract}

To any locally compact group $G$ are naturally associated two $C^{*}$-algebras: the full $C^{*}$-algebra $C^{*} G$ which contains the information on all unitary representations of $G$, and the reduced $C^{*}$ algebra $C_{r}^{*} G$ which only takes into account the unitary representations weakly contained in the regular representation in $L^{2}(G)$. There is a surjective morphism $\lambda: C^{*} G \rightarrow C_{r}^{*} G$ which is an isomorphism if and only if $G$ is amenable.

The $K$-theory functor, a covariant functor from $C^{*}$ algebras to abelian groups, gives rise to a morphism $\lambda_{*}$ of abelian groups . J. Cuntz [C] has proved that $\lambda_{*}$ is an isomorphism for some non amenable discrete groups such as free groups or $S L(2, \mathbf{Z})$. Such groups are said to be $K$-amenable. Strictly speaking, one requires a slightly stronger property: the isomorphism in $K$ theory must hold not only for the group $C^{*}$-algebras of $G$ but for the crossed products associated to the action of $G$ by automorphisms on an auxiliary $C^{*}$-algebra. In our 1983 paper [JV1], A. Valette and myself gave a generalization of J. Cuntz's result, proving K-amenability of any locally compact group acting on a tree with amenable stabilizers. The

Received by the editors in June 2014 - In revised form in September 2014.

Communicated by A. Valette.

2010 Mathematics Subject Classification : 19K35, 19K99, 46L80, 22D10, 22 D12.

Key words and phrases : K-theory, Operator Algebras, Group Representation Theory. 
most striking example was the (totally disconnected) group $S L(2)$ over the field of $p$-adic numbers. Details are given in [JV2].

The technical tool, both in Cuntz's paper and in our work, is G. Kasparov's equivariant $K K$-bifunctor $[K]$. An important special case is the ring $K K_{G}(\mathbf{C}, \mathbf{C})$ associated to a locally compact group $G$. If $G$ is compact, this is nothing else as the representation ring $R(G)$. In general, it is defined as the set of homotopy classes of G-Fredholm modules. The existence of a product in a non trivial result in $[\mathrm{K}]$.

The only thing we shall need here is the definition of a G-Fredholm module: it is given by two unitary representations $\pi_{1}$ and $\pi_{2}$ of $G$ respectively on Hilbert spaces $H_{1}$ and $H_{1}$, together with a bounded Fredholm operator $T: H_{1} \rightarrow H_{2}$ such that $T \pi_{1}(g)-\pi_{2}(g) T$ is compact for any $g \in G$ and depends on $g$ in a norm continuous manner.

The proof of [JV2] combined two ingredients:

1) The construction of a very simple Fredholm module associated to the group action on a tree. It defines a class $\gamma$ in Kasparov's ring $K K_{G}(\mathbf{C}, \mathbf{C})$.

2) The construction of a homotopy proving that $\gamma=1$ using the fact that the distance kernel on the set of vertices of the tree is of conditionally negative type. Or equivalently the existence of an affine action of $G$ on the $\ell^{2}$ space of edges of the tree.

Note that the two ingredients are of a rather different nature. The first can be generalized to other situations such as Bruhat-Tits buildings [JV3][KS1] or (hyper)bolic spaces [KS2]. The second is very specific to trees or generalizations (e.g. CAT(0) cubic complexes cf [BGH]).

I present here a perhaps more natural proof of the same result. It is inspired by the nice construction by Pytlik and Szwarc [PS] of a family of uniformly bounded representations of a free group, generalized by Valette [V1] and Szwarc [S1] to groups acting on trees. I have had this new version for quite a long time in my private notes. I thank Amaury Freslon and Jacek Brodzki for convincing me that making these notes available could be useful to others. I thank Ryszard Szwarc for pointing me reference [S1] and Jean-Pierre Schreiber for some comments and corrections. [S2].

I dedicate this short paper to the memory of Tadeusz Pytlik, who died in 2006

\section{Notations}

Let $X=\left(X^{0}, X^{1}\right)$ be a tree. There is no orientation on $X$ so that the set $X^{1}$ of edges is just a subset of $X^{0} \times X^{0}$ stable by the map $(x, y) \mapsto(y, x)$. By hypothesis, for any $x$ and $y$ in $X^{0}$ there is a unique path joining $x$ to $y$. We denote by $[x, y]$ the set of vertices lying between $x$ and $y$ and $d(x, y)$ the number of edges between $x$ and $y$.

Let $G$ be a group acting on $X$. In other words $G$ acts on $X^{0}$ in such a way that the subset $X^{1} \subset X^{0} \times X^{0}$ is stable. For simplicity we consider a discrete group $G$ but the arguments can be easily generalized to the case of a locally 
compact group $G$, such as $S L_{2}\left(\mathbf{Q}_{p}\right)$, cf. [JV2]. We consider the Hilbert space $\ell^{2}\left(X^{0}\right),\left(\delta_{x}, x \in X^{0}\right)$ its canonical Hilbert basis and $\pi_{0}$ the unitary representation arising from the action of $G$ on the set $X^{0}$ defined by $\pi_{0}(g) \delta_{x}=\delta_{g x}$. Let $\ell^{2}\left(X^{1}\right)^{-}$ be the quotient of $\ell^{2}\left(X^{1}\right)$ by the subspace generated by the vectors $\delta_{(x, y)}+\delta_{(y, x)}$ for $(x, y) \in X^{1}$ and $\pi_{1}$ the unitary representation of $G$ on $\ell^{2}\left(X^{1}\right)^{-}$defined by $\pi_{1}(g) \delta_{(x, y)}=\delta_{(g x, g y)}$

For $x \in X^{0}$ let $V(x)$ be the set of neighbours of $x$. Let the cardinality of $V(x)$ be denoted $q_{x}+1$. We assume for simplicity that $q_{x}$ is bounded. We define the following bounded operators $S$ and $Q$ on $\ell^{2}\left(X^{0}\right)$ by the following formulae:

$$
\begin{gathered}
S \delta_{x}=\sum_{y \in V(x)} \delta_{y} \\
Q \delta_{x}=q_{x} \delta_{x} .
\end{gathered}
$$

Note that the operators $Q$ and $S$ only depend on the tree structure on $X$ and therefore commute with the unitaries $\pi_{0}(g)$.

\section{The Pytlik-Szwarc operator}

We now choose an origin $x_{0} \in X^{0}$. Let $p_{0}$ the orthogonal projection onto the vector $\delta_{x_{0}}$. Let us define the operator $P$ on $\ell^{2}\left(X^{0}\right)$ by

$$
\begin{aligned}
& P \delta_{x_{0}}=0 \\
& P \delta_{x}=\delta_{x^{\prime}}
\end{aligned}
$$

where for $x \neq x_{0}$ we define $x^{\prime}$ to be the unique neighbour of $x$ lying between $x_{0}$ and $x$.

Proposition 1. The operator $P$ such defined is bounded on $\ell^{2}\left(X^{0}\right)$ and satisfies:

$$
\begin{gathered}
P P^{*}=Q+p_{0} \\
P+P^{*}=S
\end{gathered}
$$

Proof: For $x \neq x_{0}$, one has $P^{*} \delta_{x}=\sum_{y \in V(x) \backslash\left\{x^{\prime}\right\}} \delta_{y}$ so that clearly $\left(P+P^{*}\right) \delta_{x}=$ $S \delta_{x}$. Similarly $\left(P+P^{*}\right) \delta_{x_{0}}=P^{*} \delta_{x_{0}}=\sum_{y \in V\left(x_{0}\right)} \delta_{y}=S \delta_{x_{0}}$.

On the other hand, for $x \neq x_{0}$ and $y \in V(x) \backslash\left\{x^{\prime}\right\}$ one has $P \delta_{y}=\delta_{x}$ so that $P P^{*} \delta_{x}=q_{x} \delta_{x}$, whereas for any $y \in V\left(x_{0}\right), P \delta_{y}=\delta_{x_{0}}$ so that $P P^{*} \delta_{x_{0}}=\left(q_{x_{0}}+1\right) \delta_{x_{0}}$

Corollary 1. Let $T_{t}=1-t P+\left(\left(1-t^{2}\right)^{1 / 2}-1\right) p_{0}$ for any $t \in[0,1]$ Then the operator $T_{t}$ satisfies the following formula: $T_{t} T_{t}^{*}=1-t S+t^{2} Q$. In particular, the operator $T_{t} T_{t}^{*}$ commutes with the unitaries $\pi_{0}(g), g \in G$.

Indeed we have $T_{t}=1-t P+\alpha p_{0}$ where $\alpha$ satisfies $\alpha^{2}+2 \alpha+t^{2}=0$. A straightforward calculation (using the obvious fact that $P p_{0}=0$ ) yields $T_{t} T_{t}^{*}=1-t\left(P+P^{*}\right)+t^{2} P P^{*}+\left(2 \alpha+\alpha^{2}\right) p_{0}$ and the result follows from the proposition. 


\section{Construction of new representations}

Let us consider the space $D\left(X^{0}\right)$ of finitely supported functions on $X^{0}$ as a dense subspace of $\ell^{2}\left(X^{0}\right)$. Clearly $D\left(X^{0}\right)$ is stable by the operators $P, P^{*}$ and $\pi_{0}(g)$ for $g \in G$.

Lemma 1. For any complex number $z$ the operator

$$
(1-z P)^{-1}=\sum_{k=0}^{\infty} z^{k} P^{k}
$$

is defined on $D\left(X^{0}\right)$. One has

$$
(1-z P)^{-1} \delta_{x}=\sum_{y \in\left[x_{0}, x\right]} z^{d(y, x)} \delta_{y}
$$

Proof. Let the elements of $\left[x_{0}, x\right]$ be denoted $x_{0}, x_{1}, \ldots, x_{n}=x$, where $n=$ $d\left(x_{0}, x\right)$. Then $P^{k} \delta_{x}=\delta_{x_{n-k}}$ for $k \leq n$ and 0 for $k>n$. Note that $d\left(x_{n-k}, x\right)=k$. This makes the statement straightforward.

Theorem 1. (Pytlik-Szwarc) Let $z$ be a complex number such that $|z|<1$. For any $g \in G$ the operator $\rho_{z}(g)=(1-z P)^{-1} \pi_{0}(g)(1-z P)$ extends to a bounded operator on $\ell^{2}\left(X^{0}\right)$, thus defining a representation $\rho_{z}$ of $G$ in $\ell^{2}\left(X^{0}\right)$. The operator $\rho_{z}(g)-\pi_{0}(g)$ is a finite rank operator and the representation $\rho_{z}$ is uniformly bounded, i.e.

$$
\sup _{g \in G}\left\|\rho_{z}(g)\right\|<\infty
$$

In order to construct unitary representations, we shall need to modify the operators $1-t P$ by the operators $T_{t}$ as in Corollary 1 . Note that the inverse operator $T_{t}^{-1}$ is well defined on the space $D\left(X^{0}\right)$.

Theorem 2. For any real number such that $0<t<1$ the operator $\tilde{\rho}_{t}(g)=T_{t}^{-1} \pi_{0}(g) T_{t}$ on $D\left(X^{0}\right)$ extends to a unitary operator on $\ell^{2}\left(X^{0}\right)$, thus defining a unitary representation $\tilde{\rho}_{t}$ of $G$ in $\ell^{2}\left(X^{0}\right)$. The operator $\tilde{\rho}_{t}(g)-\pi_{0}(g)$ is a finite rank operator. The uniformly bounded representation $\rho_{t}$ is equivalent to the unitary representation $\tilde{\rho}_{t}$.

Proof of theorems 1 and 2: Let us prove that $\rho_{z}(g)-\pi_{0}(g)$ has finite rank and a norm bounded independently from $g$. It is enough to consider the operator

$$
\rho_{z}(g) \pi_{0}(g)^{-1}-1=z(1-z P)^{-1}\left(P-P^{\prime}\right)
$$

where $P^{\prime}$ is defined just as $P$ but replacing $x_{0}$ by $g x_{0}$. It is clear that the above operator is supported on the finite dimensional subspace generated by the $\delta_{x}$ 's for $x \in\left[x_{0}, g x_{0}\right]$. That subspace is indeed stable by $P$ and $P^{\prime}$ which have restrictions of norm 1 . Therefore the norm of $(1-z P)^{-1}\left(P-P^{\prime}\right)$ is at most $2 \sum|z|^{k}=$ $2(1-|z|)^{-1}$. This proves theorem 1 . 
To deduce theorem 2 note that $\tilde{\rho}_{t}(g)=u_{t}^{-1} \rho_{t}(g) u_{t}$ where $u_{t}=\left(1-p_{0}\right)+$ $\left(1-t^{2}\right)^{1 / 2} p_{0}$ is an invertible operator which differs from the identity by a compact operator. It remains to show that $\tilde{\rho}_{t}(g)$ is unitary. Since it is invertible it is enough to compute $\tilde{\rho}_{t}(g)^{*} \tilde{\rho}_{t}(g)=T_{t}^{*} \pi_{0}(g)^{-1}\left(T_{t} T_{t}^{*}\right)^{-1} \pi_{0}(g) T_{t}$ which is equal to 1 since $T_{t} T_{t}^{*}$ commutes to $\pi_{0}(g)$ by the corollary to the proposition above.

Remark. The link with the original approach of [JV1][JV2] is given by an easy computation: the kernel defining the (densely defined) operator $\left(T_{t} T_{t}^{*}\right)^{-1}$ is

$$
<T_{t}^{-1} \delta_{x}, T_{t}^{-1} \delta_{y}>=t^{d(x, y)}=e^{-\lambda d(x, y)}
$$

if $t=e^{-\lambda}$.

\section{The limit when $t$ tends to 1}

Let us now calculate the limit of $\tilde{\rho}_{t}(g)$ when $t \rightarrow 1$. Let us recall the definition of the Julg-Valette map $F: \ell^{2}\left(X^{0}\right) \rightarrow \ell^{2}\left(X^{1}\right)^{-}$:

$$
\begin{gathered}
F \delta_{x_{0}}=0 \\
F \delta_{x}=\delta_{\left(x, x^{\prime}\right)}
\end{gathered}
$$

We have $F p_{0}=0, F^{*} F=1-p_{0}$ and $F F^{*}=1$.

Lemma 2. Let $b: \ell^{2}\left(X^{1}\right)^{-} \rightarrow \ell^{2}\left(X^{0}\right)$ be the coboundary operator defined by $b \delta_{(x, y)}=$ $\delta_{y}-\delta_{x}$. Then the operators $F$ and $P$ are related by the formulae:

$$
\begin{gathered}
1-P=b F+p_{0} \\
(1-P) F^{*}=b
\end{gathered}
$$

Indeed, $(1-P) \delta_{x}=\delta_{x}-\delta_{x^{\prime}}=b F \delta_{x}$ if $x \neq x_{0}$ and $(1-P) \delta_{x_{0}}=\delta_{x_{0}}$. The second formula follows from the first since $(1-P) F^{*}=b F F^{*}=b$.

Remark. Let $c(x, y)=\sum \delta_{\left(x_{i}, x_{i+1}\right)}$ if the elements of $[x, y]$ are denoted $x_{0}=x$, $x_{1}, \ldots, x_{n}=y$. This is the cocycle realizing explicitly the Haagerup property for groups acting properly on trees: $\|c(x, y)\|^{2}=d(x, y)$. On has $b c(x, y)=\delta_{y}-\delta_{x}$, hence by the second formula above, $c(x, y)=F(1-P)^{-1}\left(\delta_{y}-\delta_{x}\right)$.

Corollary 2. The operator $(1-P)^{-1} b$ extends to a bounded operator and one has:

$$
(1-P)^{-1} b=F^{*}
$$

It follows indeed from the lemma that $(1-P) F^{*}=b$.

Proposition 2. For any $g \in G$ the unitary operator $\tilde{\rho}_{t}(g)(0<t<1)$ converges strongly to

$$
\tilde{\rho}_{1}(g)=F^{*} \pi_{1}(g) F+p_{0}
$$

when $t \rightarrow 1$. 
Proof: Let us first prove that $F \tilde{\rho}_{t}(g) F^{*}$ strongly converges to $\pi_{1}(g)$. One has (as $\left.p_{0} F^{*}=0\right)$ :

$$
F \tilde{\rho}_{t}(g) F^{*}=F T_{t}^{-1} \pi_{0}(g) T_{t} F^{*}=F(1-t P)^{-1} \pi_{0}(g)(1-t P) F^{*}
$$

which evaluated on functions with finite support on $X^{1}$ converges to $F(1-P)^{-1} \pi_{0}(g)(1-P) F^{*}$, but this is equal to

$$
F(1-P)^{-1} \pi_{0}(g) b F F^{*}=F(1-P)^{-1} b \pi_{1}(g)=F F^{*} \pi_{1}(g)=\pi_{1}(g)
$$

by two applications of Corollary 2.

We deduce that $\left(1-p_{0}\right) \tilde{\rho}_{t}(g)\left(1-p_{0}\right)$ converges strongly to $F^{*} \pi_{1}(g) F$.

On the other hand we check that $\tilde{\rho}_{t}(g) \delta_{x_{0}} \rightarrow \delta_{x_{0}}$ when $t \rightarrow 1$. We have indeed $T_{t} \delta_{x_{0}}=\left(1-t^{2}\right)^{1 / 2} \delta_{x_{0}}$ so that $(1-t P)^{-1} \pi_{0}(g) T_{t} \delta_{x_{0}}=\left(1-t^{2}\right)^{1 / 2}(1-t P)^{-1} \delta_{g x_{0}}=$ $\left(1-t^{2}\right)^{1 / 2} \sum t^{d\left(y, g x_{0}\right)} \delta_{y}$ where the sum is extended to the vertices $y$ of the segment $\left[x_{0}, g x_{0}\right]$. Finally $T_{t}^{-1} \pi_{0}(g) T_{t} \delta_{x_{0}}=t^{d\left(x_{0}, g x_{0}\right)} \delta_{x_{0}}+\left(1-t^{2}\right)^{1 / 2} \sum t^{d\left(y, g x_{0}\right)} \delta_{y}$ where $x_{0}$ is now excluded from the sum. Hence the result.

As a consequence $\tilde{\rho}_{t}(g) p_{0}$ converges normally to $p_{0}$, and since $\tilde{\rho}_{t}$ is unitary, replacing $g$ by $g^{-1}$ we also have that $p_{0} \tilde{\rho}_{t}(g)$ converges normally to $p_{0}$.

The proposition clearly follows.

\section{Classes in $K K_{G}$-theory}

Let us consider the Hilbert space $\ell^{2}\left(X^{0}\right)$ equipped with the two representations $\tilde{\rho}_{t}$ and $\pi_{0}$, which differ by compact operators. The triple $\left(\tilde{\rho}_{t}, \pi_{0}, I d\right)$ defines an element of the Kasparov group $K K_{G}(\mathbf{C}, \mathbf{C})$. It is independent of the value on $t \in[0,1]$ since the operators $\tilde{\rho}_{t}(g)$ are strongly continuous in $t$. Now when $t=0$ we have $\tilde{\rho}_{0}=\pi_{0}$ so that the element is equal to 0 . On the other hand when $t=1$ we have $\tilde{\rho}_{1}(g)=F^{*} \pi_{1}(g) F+p_{0}$ so that the element is equal to $1-\gamma$ where $\gamma$ is defined as in [JV1][JV2] by the triple $\left(\pi_{0}, \pi_{1}, F\right)$.

Corollary 3. We have the equality $\gamma=1$ in the Kasparov group $K K_{G}(\mathbf{C}, \mathbf{C})$.

Recall [JV2] that the above corollary implies the $K$-theoretic amenability of $G$ under the hypothesis that the vertices of the tree have amenable stabilisers.

\section{References}

[BGH] Jacek Brodzki, Erik Guentner, Nigel Higson A differential complex for groups acting on $\mathrm{CAT}(0)$ cube complex, unpublished.

[C] Joachim Cuntz, K-theoretic amenability for discrete groups, J. Reine Angew. Math. 344 (1983), 180-195.

[JV1] Pierre Julg, Alain Valette, K-moyennabilité pour les groupes opérant sur les arbres, C. R. Acad. Sci. Paris Sér. I Math. 296 (1983), no. 23, 977-980. 
A new look at the proof of K-theoretic amenability for groups acting on trees 269

[JV2] Pierre Julg, Alain Valette,K-theoretic amenability for $S L_{2}\left(\mathbf{Q}_{p}\right)$, and the action on the associated tree, J. Funct. Anal. 58 (1984), no. 2, 194-215.

[JV3] Pierre Julg, Alain Valette, Fredholm modules associated to Bruhat-Tits buildings, Miniconferences on harmonic analysis and operator algebras (Canberra, 1987), 143-155, Proc. Centre Math. Anal. Austral. Nat. Univ., 16, Austral. Nat. Univ., Canberra, 1988.

[K] Gennadi Kasparov, Equivariant KK-theory and the Novikov conjecture, Invent. Math. 91 (1988), n0 1, 147-201.

[KS1] Gennadi Kasparov, Georges Skandalis, Groups acting on buildings, operator K-theory, and Novikov's conjecture, K-Theory 4 (1991), no. 4, 303-337.

[KS2] Gennadi Kasparov, Georges Skandalis, Groups acting properly on "bolic" spaces and the Novikov conjecture. Ann. of Math. (2) 158 (2003), no. 1, 165-206.

[PS] Tadeusz Pytlik, Ryszard Szwarc, An analytic family of uniformly bounded representations of free groups, Acta Math. 157 (1986), no. 3-4, 287-309.

[S1] Ryszard Szwarc, Groups acting on trees and approximation properties of the Fourier algebra, J. Funct. Anal 95 (1991), 320-343.

[S2] Ryszard Szwarc, Tadek Pytlik in my memories, Colloquium Mathematicum, 108 (2007), no. 1, 1-6.

[V1] Alain Valette, Cocycles d'arbres et représentations uniformément bornées. C. R. Acad. Sci. Paris Sér. I Math. 310 (1990), no. 10, 703-708.

[V2] Alain Valette, Les représentations uniformément bornées associées un arbre réel, Bull. Soc. Math. Belg. Sér. A 42 (1990), no. 3, 747-760.

[V3] Alain Valette, Addendum: "Uniformly bounded representations associated with a real tree". Bull. Soc. Math. Belg. Sér. A 44 (1992), no. 1, 101-102.

MAPMO (Université d'Orléans et CNRS)

Bâtiment de Mathématiques - Rue de Chartres

B.P. 6759 - 45067 Orléans cedex 2

FRANCE 